Omur Gurel Selimoglu 1

Selda Ozdemir ${ }^{2}$

Gokhan Toret $^{3}$

Ufuk Ozkubat $^{4}$

\section{An Examination of the Views of Parents of Children With Autism About Their Experiences at the Post-Diagnosis Period of Autism}

\begin{abstract}
The purpose of this study was to examine the perspectives of parents of children with autism regarding their experiences about their children's diagnosis and services that they receive in the areas of special education, health and personal needs. The participants of the study were 50 parents who were the primary caregivers of children with autism. A qualitative research method was used in the study. Semi-structured interview questions were developed and study data were analyzed using interpretive content analysis technique. Major research findings were presented under four themes: 1) time difference between the recognition of children's developmental differences and medical diagnosis, 2) assessment limitations experienced during the clinical diagnosis, 3) lack of support services for parental needs, and 4) collaboration problems among the various special education services. The findings of the study were discussed and implications for practice were provided.
\end{abstract}

Keywords: Autism spectrum disorder, parents' opinions, diagnostic period, early intervention.

\footnotetext{
${ }^{1}$ Research Assistant, Gazi University, Faculty of Education, Department of Special Education, Ankara, Turkey E-mail: ogurel@gazi.edu.tr

${ }^{2}$ Assoc. Prof. Dr., Gazi University, Faculty of Education, Department of Special Education, Ankara, Turkey E-mail: seldaozdemir@gazi.edu.tr

${ }^{3}$ Research Assistant, Gazi University, Faculty of Education, Department of Special Education, Ankara, Turkey E-mail:gokhantoret@hotmail.com ${ }^{4}$ Research Assistant, Gazi University, Faculty of Education, Department of Special Education, Ankara, Turkey
E-mail: ozkubat@gmail.com
}

International Journal of Early Childhood Special Education (INT-JECSE), 5(2), 129-167. 


\section{Structured Abstract}

Deciding to have a family and a child is not only an important decision in a person's life but also it brings a dramatic change in a family' life circle. A new family member brings new responsibilities for parents and significant changes in family routines. In addition to the new responsibilities, life styles of the couples change and responsibilities of the couples to each other change as a result of having a new family member (Girli, 2004; Kucuker, 2001). Because of a child's needs and child caring responsibilities, having a typically developing child may increase parental stress. Parents of a child with special needs would experience more parental stress. In addition, families would experience negative feelings such as guilt, disappointment and depression as a result of having a child with special needs (Fisman, Wolf \& Noah, 1989; Oksuz, 2008; Vardarci, 2011).

In the pre-diagnostic period, when parents begin to observe strange differences in their child's development and face the diagnosis of autism, generally their perceptions regarding the new experience is an unknown feeling and a denial of the diagnosis. Moreover, many parents of children with autism worry about their increasing child caring duties related to their child' needs and potential negative effects of having a child with autism in the family life.

Early childhood special education services are designed to support children's development by establishing reciprocal interactions between early intervention service providers and parents. Providing necessary support services for families during the early childhood period and accepting the importance of parents in children's learning are very important in establishing effective support mechanisms for families and developing necessary regulations related to health and educational services. Thus, the purpose of the study was to examine the perspectives of parents of children with autism regarding their experiences about their children's diagnosis, educational and health services provided to their children and their personal needs. This study will, therefore, will address the following research questions:

1. What are the experiences of parents of children with autism in regard to their personal needs, demands and challenges encountered during their child's pre and post-diagnosis periods?

2. What are the views of families on services received in the areas of special education, medical diagnosis, and medical care?

\section{Method}

\section{Research Model}

This study has been conducted using a qualitative research design. Semi-structured interviews were conducted with the participating parents and parents' answers to questions were generated under research themes that indicate social support perceptions of the participating parents. 


\section{Participants}

The participants were identified using the criterion sampling technique which is one kind of the purposive sampling techniques. The selection criteria for generating the participating parents were as follows: The participants c) were the primary caregivers of children with autism, a) had children with autism between the ages of 2-12, b) their children received the diagnoses from child psychiatrists who work for state hospitals and/or university hospitals, and d) were willing to participate in the study. Having met the specified criteria, a total of 50 participants accepted to join the study. From fifty participants, ten had children enrolled in special education centers located in Ankara, twenty had children enrolled in official autistic children education centers located in Isparta, and twenty had children enrolled in official autistic children education centers and special education centers located in Istanbul.

\section{Data Collection}

Semi-structured interviews were conducted in the study. An interview form which included semi-structured and open-ended questions that were designed based on the literature review was developed for the interviews. Following the identification of the research questions, three researchers conducted one-on-one interviews with fifty participating parents. All interviews were conducted on the day and time that were appropriate for the participants. Each interview lasted approximately 30 to 60 minutes.

\section{Data Analysis}

Following the completion of the interviews with the parents, the interviews were transcribed without making any corrections; the expressions of parents were written verbatim as they were heard by the researchers.

Descriptive analysis of the study data was carried out using the research themes identified by the researchers in a consensus. Descriptive analyses were conducted by the researchers independently and the reliability was achieved by reaching a consensus on the analyses.

\section{Results}

The participating families $(n=33,66 \%)$ reported that they did not observe their children's any verbal communication attempts and children exhibited significant speech and language delays in the pre diagnostic period.

After asking the question about the time that they noticed the behavioral differences in their children, $24 \%$ of participating parents answered the question explaining that their children were about 1,5 years old, $24 \%$ of the parents answered that their children were about 2 years old and 17\% of the parents answered that their children were about 3 years old, when they first observed some strange behaviors and developmental delays in their children.

Parents explained that once they begin to observe some developmental delays and unexpected behaviors, they began to diagnostic evaluations by taking their children to a 
doctor. The mean diagnostic ages among children were: $24 \%$ of children were about 4 years old, $20 \%$ of children were about 2,5 years old, and $14 \%$ of children were about 3 years old.

$64 \%$ of the families reported that they were not informed about autism, $36 \%$ of families reported that they were not informed about educational and health related services available to them, and $68 \%$ of families reported that they were not informed about the difficulties that they would face in their lifetime.

$46 \%$ of the families criticized that they were not provided with any kinds of information and guidance related to their children's diagnosis. In addition, $36 \%$ of the families reported that they do not receive appropriate health care services and complained about the inappropriate behaviors and negative attitudes of some hospital staff.

$88 \%$ of the participating families stated that they have psychological needs, $86 \%$ of the families stated that they need more information about autism and related issues, $80 \%$ of the families stated that they have educational needs and $74 \%$ of the families stated that they need financial support services.

One of the major research findings was that participating families made personal efforts to address their personal, child care and educational needs. While $44 \%$ of these families reported that they tried to address their information needs by using internet to access more information about autism, $24 \%$ of the parents stated that they tried to get more information on autism by reading books on autism and $42 \%$ of the families reported that they tried to address their psychological needs by going to psychologists and specialists working in private special education centers where their children receive education.

$36 \%$ of the families reported some dissatisfaction with the education provided to their children and reported that their children's functional living skills were not addressed in the schools and educational sessions provided to their children were not enough. $50 \%$ of these families stated that retired or incompetent teachers work with their children in private special education centers.

\section{Discussion}

Findings of this study yielded four several important outcomes: 1) time difference between the recognition of children's developmental difference and medical diagnosis, 2) assessment limitations experienced during the clinical diagnosis 3) the lack of support services for parental needs, and 4) collaboration problems of special education services.

The diagnostic criteria for autism spectrum disorders reflect developmental problems including, but not limited to, three core-defining features: impairments in socialization, impairments in verbal and non-verbal communication, and restricted and repetitive patterns of behaviors (American Psychiatric Association, 1994). As autism symptoms and characteristics can present themselves in a wide variety of combinations from mild 
to severe, it can be very difficult for parents to notice their children' atypical behaviors and development delays. Experienced child psychiatrists rely on information gathered from multiple sources. Recognizing autism in an early age and proving children with necessary early intervention services are now possible in many developed countries.

The participating families reported that during their child's diagnostic evaluation period, child psychiatrists and neurologists used only parental information about their child's behaviors and development and the evaluation procedure did not include any observations of the child's behaviors. Furthermore, these families reported that the evaluations were administered in a very short time. Thus, the problems experienced by the participating parents during diagnostic evaluation process affected negatively these parents' acceptance of their child's diagnosis.

Early indicators of autism and especially social interactional cues of autism must be well-recognized by the child psychiatrists and a variety of evaluation tools and procedures should be administered to gather relevant functional and developmental information about a child's functioning. It is well known that social interactional difficulties displayed by children with autism are very unique and distinctive characteristics of the autism (Moolman-Smok, Vertomer, Buckle, \& Linderberg, 2008). The evaluation of autism must rely on conducting systematic observations and family reports about child's functional and developmental skills. Procedures used in the evaluation process will lead parents to trust the diagnosis and such procedure will enable parents to both recognize and accept their child's diagnosis.

In addition, after the diagnosis of autism, the person that gives the first information about the diagnosis to parents and the way that the information transferred to families become very important. The information provided to families affects parents' later adaptation to their child's diagnosis. In addition, in order to achieve better parental coping, it is important to establish positive, caring, and mutual relationships among professional, parents, and children. The service providers have a key role in the administration of the entire diagnosis and early intervention process. With the support of professionals, families can learn, develop, and implement new skills to support their children's development, can search for assistance when needed, and reach information when necessary. Although early intervention practices are based on supporting children's development and diminishing children's developmental risks, establishing reciprocal interactions between early intervention service providers and parents is very important. Even though special education services require parents' active participation in the educational practices implemented in Turkey (Ministry of National Education, 1997), most of the early intervention practices focus on child-centered approaches rather than parent-centered practices (Ozdemir, 2008). As discussed earlier, education programmes and related services provided to children with special needs and their families must be reevaluated in Turkey. Because of the importance of parental coping skills, family-centered support services must be effectively designed and provided to families of children with autism. Based on the findings gathered in this study, it can be concluded that there is an urgent need in establishing effective support mechanisms and 
implementing necessary regulations in health and educational services in Turkey in order to address the needs of children with autism and their parents. 